\title{
Vivencias socioemocionales en familias de jóvenes con Síndrome de Down en Padre Las Casas, Chile
}

Socio-emotional experiences in families of young people with Down

Syndrome in Padre Las Casas, Chile

\section{Volumen 19, Número 3 \\ Setiembre-Diciembre}

pp. 1-26

\author{
Yessica Antisoli Remolcoy \\ Claudia Daniela Campusano Toledo \\ Teresa Maricel Moraga Leiva \\ María José Uberuaga Henríquez \\ Daniel San Martín Cantero \\ Carmen Paz Tapia Gutiérrez
}

\section{Citar este documento según modelo APA}

Antisoli Remolcoy, Yessica., Campusano Toledo, Claudia Daniela., Moraga Leiva, Teresa Maricel., Uberuaga Henríquez, María José., San Martín Cantero, Daniel. y Tapia Gutiérrez, Carmen Paz. (2019). Vivencias socioemocionales en familias de jóvenes con Síndrome de Down en Padre Las Casas, Chile. Revista Actualidades Investigativas en Educación, 19(3), 1-26. Doi. 10.15517/aie.v19i3.38783 


\title{
Vivencias socioemocionales en familias de jóvenes con Síndrome de Down en Padre Las Casas, Chile
} Socio-emotional experiences in families of young people with Down Syndrome in Padre Las Casas, Chile

\author{
Yessica Antisoli Remolcoy ${ }^{1}$ \\ Claudia Daniela Campusano Toledo ${ }^{2}$ \\ Teresa Maricel Moraga Leiva ${ }^{3}$ \\ María José Uberuaga Henríquez \\ Daniel San Martín Cantero 5 \\ Carmen Paz Tapia Gutiérrez ${ }^{6}$
}

\begin{abstract}
Resumen: Este artículo tiene como objetivo mostrar las vivencias socioemocionales de familias con hijos e hijas que presentan Síndrome de Down. Se trata de una investigación realizada durante el año 2018, en la que se abordan relaciones sociales y etapas emocionales que reestructuran el curso de vida de familias. El enfoque metodológico es cualitativo, con un diseño de estudio de caso. Las personas participantes fueron integrantes de familias de jóvenes con Síndrome de Down, personas integrantes de una agrupación para familias de hijos o hijas con Síndrome de Down en la comuna de Padre las Casas en Chile. Las técnicas para la recolección de información fueron semblanzas y entrevistas biográficas-narrativas. Se realiza análisis inductivo-deductivo, y se aplica la triangulación de datos. Los resultados se organizaron en torno a cinco ejes temáticos: (i) recibir la noticia, (ii) apoyo socio-familiar, (iii) experiencias con escuelas, (iv) resiliencia, y (v) curso emocional. Se concluye que las vivencias socioemocionales condicionan el rumbo de vida de las familias. Las significatividades interpretadas mostraron ser útiles para comprender el rol de instituciones de salud, educativas y sociales, y su aporte en procesos de aprendizaje, desarrollo y acompañamiento del niño, niña y su familia.
\end{abstract}

Palabras clave: vivencias socioemocionales, familia, síndrome de Down

\footnotetext{
1 Estudiante de Pedagogía en Educación Diferencial, Universidad Católica de Temuco, Chile. Dirección electrónica: yantisoli2015@alu.uct.cl

2 Estudiante de Pedagogía en Educación Diferencial, Universidad Católica de Temuco, Chile. Dirección electrónica: ccampusano2015@alu.uct.cl

3 Estudiante de Pedagogía en Educación Diferencial, Universidad Católica de Temuco, Chile. Dirección electrónica: tmoraga2015@alu.uct.cl

4 Estudiante de Pedagogía en Educación Diferencial, Universidad Católica de Temuco, Chile. Dirección electrónica: muberuaga2015@alu.uct.cl

${ }^{5}$ Académico de la Universidad Católica de Temuco, Director del Centro de Investigación Escolar y Desarrollo, Facultad de Educación, Chile. Dirección electrónica:dsanmartin@uct.cl

6 Académica de la Universidad Católica de Temuco en la Carrera Educación Diferencial, Departamento de Infancia y Educación Básica, Facultad de Educación, Chile. Dirección electrónica: ctapia@uct.cl
}

Artículo recibido: 29 de marzo, 2019

Enviado a corrección: 4 de junio, 2019

Aprobado: 15 de julio, 2019 


\begin{abstract}
This article aims to show the socio-emotional experiences of families with sons and daughters who have Down Syndrome. This is an investigation carried out during the year 2018, in which social relationships and emotional stages that restructure the life course of families are addressed. The methodological approach is qualitative, with a case study design. The participants were members of families of young people with Down Syndrome, members of a group for families of children with Down Syndrome in the commune of Padre Las Casas in Chile. The techniques for gathering information were semblances and biographical-narrative interviews. Inductive-deductive analysis is performed, and data triangulation is applied. The results were organized around five thematic axes: (i) receiving the news, (ii) socio-family support, (iii) experiences with schools, (iv) resilience, and $(v)$ emotional course. It is concluded that socio-emotional experiences determine the life course of families. The interpreted significance showed to be useful to understand the role of health, educational and social institutions, and their contribution in learning processes, development and accompaniment of the boy, girl and their family.
\end{abstract}

Keywords: socio-emotional experiences, family, Down's syndrome

\title{
1. Introducción
}

Esta investigación centra su atención en la familia, considerada como un pilar fundamental dentro de la sociedad debido a su importancia en el desarrollo de sus hijos e hijas. Urbano y Yuni (2008) señalan que la familia es el sostén que necesitan las personas en situación de discapacidad a lo largo de toda la vida. Es la familia quien defiende los derechos y satisfacen las necesidades de sus hijos e hijas. En este contexto, cuando una familia se ve enfrentada a situaciones inesperadas, como es el nacimiento de un hijo o hija con Síndrome de Down (en adelante SD), se desestabiliza y comienza un proceso de duelo que supone dolor y pena por haber perdido un ideal de bebé. Esta desestabilización constituye el paso a una reorganización emocional que implica etapas de impacto, negación, tristeza, adaptación y reorganización.

En revisiones de investigaciones sobre la experiencia de familias de niños y niñas con $\mathrm{SD}$, se evidencian lagunas en torno a esta temática. Estas lagunas, entre otras razones, se deben a la escasa atención prestada a las vivencias de familias de personas con SD (Hodapp, 2008). En efecto, se auscultan aspectos socioemocionales de las personas integrantes de la familia, por cuanto se observa solo a la persona con SD (Huerta y Rivera, 2017; Romero y Peralta, 2012). Esto se debe a un enfoque biomédico en atención temprana, que centra su interés en rasgos psicosociales del individuo con SD. Sin embargo, paulatinamente se ha avanzado hacia un modelo centrado en la familia, que enfatiza el estudio en dinámicas de poder, autonomía y participación de la familia durante el proceso de apoyo (McWilliam, 2016; Tapia, Palma y González, 2017).

Aun cuando padres y madres son responsables de proteger y cautelar el bienestar de sus hijos e hijas, desde 2009, existe en Chile el Sistema de Protección Integral a la Infancia 
(SIPI), amparado por la ley 20.379. Mediante este sistema se provee de servicios y prestaciones desde la gestación hasta los 9 años de edad. Junto a este programa, se agrega el Centro Universidad Católica SD, que distribuye en unidades de maternidad de hospitales del Servicio de Salud Pública, un Manual de Atención Temprana para niños y niñas con $S D$. Este manual es entregado a las familias como una herramienta de información inicial para comprender la Atención Temprana y la importancia de poner en práctica estrategias para el desarrollo del vínculo y estimulación temprana.

Por lo anterior, es relevante conocer las vivencias de padres y madres con hijos o hijas con SD. En particular, este estudio centra su interés sobre la experiencia socioemocional de la familia, que se inicia al recibir la noticia del nacimiento de un hijo e hija con SD. En consecuencia, la pregunta de investigación refiere a: ¿cuáles son las vivencias socio-emocionales que experimentan las familias con hijos e hijas con SD?. Asimismo, el propósito es indagar sobre aquellas vivencias socio-emocionales que transgreden la vida cotidiana de las familias a partir del momento en que padres y madres reciben la noticiadiagnóstico. En particular, se trata de vivencias socio-emocionales que derivan del apoyo familiar, organizaciones sociales, instituciones educativas y de salud.

\section{Referentes teóricos}

\subsection{Importancia de la familia}

Desde la perspectiva de los derechos humanos, la familia es reconocida como unidad natural y fundamental de la sociedad, en consecuencia debe ser protegida tanto por el Estado como por la sociedad. En efecto, la constitución chilena reconoce en la familia el núcleo fundamental de la sociedad. Por tanto, es deber del Estado proteger y fortalecer la familia (Abramovich, 2006). Así, la familia cuenta con una estructura biológica, emocional, intelectual y social que propicia un espacio socializador ideal a niños y niñas (Grau Rubio, 2013). Esta estructura genera redes de interacciones físicas, afectivas y de cuidado entre sus miembros. De acuerdo con la Convención sobre los Derechos de las Personas con Discapacidad, promulgada por la Organización de las Naciones Unidas (ONU) (2006) y ratificada en Chile en el año 2008, la familia es la unidad colectiva natural y fundamental de la sociedad. En este sentido, la familia tiene derecho a recibir protección del Estado y la sociedad, quienes de esta manera deben garantizar y contribuir el desarrollo de personas con discapacidad. 


\subsection{Etapas emocionales que vive la familia}

Al interior de la familia cada miembro tiene un rol. En el caso de las madres y padres, son responsables de la protección de sus hijos e hijas a través de cautelar el cumplimiento de derechos y necesidades básicas que requieren para su desarrollo. Ahora bien, este sistema de cuidado hacia el hijo o hija, puede ser alterado por acontecimientos que suponen una transformación de la familia (Tabla 1). Por ejemplo, el modo en que se recibe la noticia acerca de una condición física o cognitiva que trae consigo un hijo o hija al nacer. Muchas veces este tipo de noticias se entrega de formas que no contribuyen al bienestar psicosocial de la familia. En efecto, se emplea un lenguaje frío y peyorativo, desde la discapacidad sin solución y/o desde la exclusión social. Este modo de comunicar una noticia sensible interfiere en los sentimientos experimentados por familiares y obstaculiza la vinculación padre/ madre e hijo/hija (Bastidas y Alcaraz, 2011; Darezzo y Dupas, 2011).

\section{Tabla 1}

Etapas emocionales de padres y madres frente al nacimiento de un hijo o hija con SD

\begin{tabular}{ll}
\hline Etapa & \multicolumn{1}{c}{ Descripción } \\
\hline Impacto & La noticia paraliza a la familia, no comprenden la situación; escuchan y no \\
& oyen, ven y no creen. Es un sentimiento de shock que puede durar segundos \\
Negación & o continuar intermitentemente varios días. \\
& Padres y madres se resisten a la idea de la situación de su hijo o hija, por lo \\
& cual generan mecanismos de defensa; 'este no es mi hijo', 'debe ser un error', \\
& '¿por qué a mí?', '¿cómo ha podido pasar?'. Se trata de un escape \\
& momentáneo para enfrentar sentimientos de culpa. Los prejuicios y opiniones \\
Tristeza o dolor & Le terceros cobran importancia. \\
& Las personas progenitoras toman conciencia de la realidad e intentan \\
& comprender lo que ocurre. Se consolida la imagen de pérdida del bebé ideal, \\
& de los sueños destruidos y la esperanza ausente. Aparecen sentimientos de \\
& dolor, angustia y tristeza. En este período se altera la cotidianidad de la \\
Adaptación & familia. Es una fase que prepara para la aceptación. \\
& Aceptación de sentimientos propios y la situación del hijo o hija. Surge interés \\
& por aprender y conocer en detalle el SD. Hay tranquilidad consigo mismos; \\
& ven al hijo o hija como alguien con fortalezas y debilidades. La etapa de \\
& aceptación es dinámica, y no se cierra. A medida que el niño o niña crece \\
Reorganización & aparecen situaciones nuevas que requieren nueva adaptación. \\
& El recuerdo del dolor, ansiedad e impotencia quedan atrás. Los padres y \\
& madres asumen una postura diferente. La o el bebé es integrado a la familia, y \\
& el vínculo se fortalece cada día.
\end{tabular}

Fuente: Elaboración propia (2018), a partir de Rossel (2004); Ruiz, Álvarez, Arce, Palazuelos y Schelstraete, (2009); Romero y Peralta (2012); Villavicencio, Romero, Criollo y Peñaloza (2018). 
De acuerdo con lo expresado por Romero y Peralta (2012), en las etapas emocionales descritas "no hay un orden predeterminado de aparición de estos sentimientos y reacciones, puede que no se experimenten todos ellos, la intensidad de los mismos variará considerablemente con el tiempo" (p.72). En la medida en que padres y madres logran equilibrar sus emociones, estarán preparados para atender de manera pertinente las necesidades de sus hijos e hijas. Así, cuanto menos tarden en adaptarse a su nueva realidad más pronto comenzarán a trabajar en ello. Por esto "[...]tenemos que saber que estos sentimientos son totalmente normales, no son unos padres raros por tenerlos, ni mucho menos son malos padres, es lógico que las personas tengan estas reacciones inicialmente" (Down España, 2012, p.16).

A medida que la familia se reestructura, surgen nuevas inquietudes relacionadas con el futuro de sus hijos e hijas, por ejemplo, la incertidumbre sobre quién asumirá el rol de cuidador o cuidadora cuando los progenitores ya no estén presentes. En consecuencia, otros hijos e hijas pasan a ser los potenciales responsables de sus hermanos o hermanas. Por su parte, Flórez (2016) señala que “...si existe un hermano (...) tiene una responsabilidad que no puede eludir, y de nosotros depende en buena parte que esa responsabilidad haya sido cultivada de manera natural y armoniosa" (p.20).

Las familias muestran que las dificultades surgen de demandas cotidianas, relacionadas con ser padres y madres de un hijo o una hija con discapacidad. Depresión, estigma social y otros conflictos interpersonales. Sin embargo, en medio de estas dificultades también emergen valores como: optimismo, comprensión, solidaridad y búsqueda de sentido a su experiencia en un contexto de construcción de fortalezas para enfrentar esta situación. Ciertamente, el sentimiento de fortaleza es importante para el desarrollo de resiliencia, que en definitiva facilita la vivencia de emociones y sensaciones negativas (Grau Rubio, 2013). En familias con un hijo o hija con SD, la resiliencia es la respuesta ante temores y sentimientos de vulnerabilidad, puesto que permite a las familias sobreponerse a episodios de dolor emocional, y al mismo tiempo lidiar con los prejuicios de una sociedad hostil (Naranjo, 2010; Ocampo y Vargas, 2018).

\subsection{Apoyo social}

Es indispensable un 'acompañamiento y apoyo social' para las familias, es decir que puedan experimentar diversas ayudas desde la comunidad como desde instituciones especializadas. Este apoyo se puede traducir en apoyo humano y/o material, que permita a 
las familias sentirse acompañadas junto a sus hijos e hijas. Giné, Gràcia, Vilaseca y BalcellsBalcells (2009) sostienen que las familias no existen independientemente de su entorno; forman parte de un sistema social. Esto significa que la familia se encuentra en constante interacción con otros; de esta manera, si las experiencias son obstaculizadoras 0 potenciadoras influyen en el modo en que los padres y madres enfrentan nuevos retos referidos a la crianza de sus hijos e hijas.

De acuerdo con Benítez (2015), el apoyo social puede influir positivamente sobre el bienestar de familias con personas con SD a través de sentimientos de seguridad, contacto social, pertenencia, estima y afecto. Instituciones de área de salud, educación, y organizaciones sociales, con su aporte pueden contribuir a la tranquilidad de las familias.

Por ejemplo, la escuela es una red de apoyo en constante interacción con la familia. En este sentido, en Chile, la Ley general de Educación, en su artículo 4 indica que padres y madres tienen el derecho y el deber de educar a sus hijos e hijas (Ministerio de Educación, 2009). De este modo, el Estado debe garantizar un vínculo Familia-Escuela con el propósito de proteger el derecho a una educación pertinente para niños y niñas. Para hacer efectivo este derecho se promulga la Ley de Inclusión (Ministerio de Educación, 2015), con el fin de asegurar a todas las personas una educación inclusiva y de calidad. De acuerdo con esta ley, es deber del Estado incentivar que se generen "[...] las condiciones necesarias para el acceso y permanencia de los estudiantes con necesidades educativas especiales en establecimientos de educación regular o especial, según sea el interés superior del niño" (Art. 1, p.2). En Chile, niños y niñas ingresan al sistema escolar a los 4 años, y se mantienen por un periodo (pre-escolar, primaria y secundaria) de 14 años. En este periodo escolar las relaciones de familias con diversos profesionales de la educación deben basarse en la cooperación. Esto significa trabajo coordinado, colaborativo y consciente de todas las partes que participan en el proceso de enseñanza y aprendizaje (Soto e Hinojo, 2004).

Desde el área comunitaria, los antecedentes indican que la presencia de agrupaciones sociales constituye fuentes de apoyo emocional para padres y madres de hijos e hijas con SD. Según España Down (2012), en estas agrupaciones los padres y madres se comprometen y aprenden a potenciar la educación de sus hijos e hijas. Precisamente, la participación en organizaciones permite a las familias intercambiar experiencias, ser escuchadas y comprendidas, y adquirir herramientas para fortalecer el desarrollo de sus hijos e hijas. La organización social en tanto red de apoyo a la familia, reconforta y posibilita el contacto con vivencias semejantes, pero también gestiona el 
apoyo profesional, social y espiritual adecuado en momentos de dificultades (Darezzo y Dupas, 2011).

\section{Metodología}

\subsection{Enfoque}

Este estudio responde a un paradigma hermenéutico, Saavedra (2005) señala que es una estrategia que "ofrece la posibilidad de develar sentidos encubiertos que, al salir a la luz, permiten una mejor comprensión de las personas estudiadas" (p.52). El enfoque es cualitativo, que, de acuerdo con Blasco y Pérez (2007), "estudia la realidad en su contexto natural tal y como sucede sacando e interpretando los fenómenos de acuerdo con las personas implicadas" (p.17). Desde este marco metodológico, el propósito de esta investigación fue indagar en las vivencias socioemocionales de familias con hijos e hijas que tienen SD. La investigación se sustentó en el estudio de casos que, según Simons (2011), tiene como finalidad "investigar la particular, la unicidad, del caso singular (...), pero la tarea fundamental es entender la naturaleza distintiva del caso particular" (p.20). De esta manera, al realizar un análisis profundo de las vivencias de las familias, surgen aspectos comunes que dan cuenta de significatividades interpretativas que emergen de quienes participaron.

\subsection{Participantes}

Para la selección de participantes se utilizó un muestreo de tipo intencional. Este tipo de muestreo se rige por el juicio experto de personas investigadoras. Es decir, se trata de cautelar que las personas cuenten con conocimientos y experiencias suficientes para profundizar en las temáticas de la investigación. De tal modo, el muestreo intencional “...permite seleccionar casos característicos de una población limitando la muestra sólo a estos casos. Se utiliza en escenarios en las que la población es muy variable y consiguientemente la muestra es muy pequeña" (Otzen y Manterola, 2017, p. 230). En una primera instancia, la totalidad de participantes de la agrupación acceden de manera voluntaria a relatar por escrito sus vivencias mediante la elaboración de semblanzas. En una segunda instancia, se revisaron las semblanzas para determinar la riqueza de los relatos expresados. Esto permitió seleccionar a cinco participantes, quienes además accedieron a participar de una entrevista en profundidad. En consecuencia, las personas que configuraron los casos fueron un padre, tres madres y una abuela cuyas edades fluctúan entre 56 y 71 años, pertenecientes de la Agrupación PLC Down de la comuna de 
Padre las Casas, región de la Araucanía, Chile (Tabla 2). Respecto a los criterios de inclusión, se consideró a familias con hijos e hijas con SD desde 15 años de edad en adelante.

Tabla 2

Familias participantes pertenecientes a la agrupación PLC Down de la comuna de Padre Las Casas, Araucanía-Chile 2018

\begin{tabular}{llll}
\hline $\begin{array}{l}\text { Casos } \\
\text { participantes }\end{array}$ & Comuna, sector de residencia & Edad participantes & $\begin{array}{l}\text { Edad hijo } \\
\text { hija }\end{array}$ \\
\hline $\begin{array}{l}\text { Familia 1 } \\
\text { Padre y madre }\end{array}$ & Padre las Casas, sector rural & $\begin{array}{l}\text { Madre, 56 años } \\
\text { Padre, 56 años }\end{array}$ & 16 años \\
\hline $\begin{array}{l}\text { Familia 2 } \\
\text { Abuela }\end{array}$ & Padre las Casas, sector urbano & 64 años & 17 años \\
\hline $\begin{array}{l}\text { Familia 3 } \\
\text { Madre }\end{array}$ & Padre las Casas, sector urbano & 61 años & 22 años \\
\hline $\begin{array}{l}\text { Familia 4 } \\
\text { Madre }\end{array}$ & Padre las Casas, sector urbano & 71 años & 29 años \\
\hline
\end{tabular}

Fuente: Elaboración propia, 2018.

\subsection{Técnicas de recolección}

Para la recolección de la información se utilizaron dos instrumentos. El primero, una semblanza (autobiografía breve) que consiste en una narración de la propia vida, contada por el/la protagonista (Pujadas, 2002). Esta semblanza permitió conocer las vivencias de familias por medio de su relato escrito. El segundo instrumento fue una entrevista en profundidad, entendida como una “...técnica de obtener información, mediante una conversación profesional con una o varias personas para un estudio analítico de la información" (Ruiz, 2012, p.165). Esta entrevista profundizó en las vivencias socioemocionales que cada familia expresó en la semblanza. Así, el proceso de aplicación y análisis de las técnicas descritas se organizó en cuatro fases según se indica en la Figura 1. 
Figura 1

Proceso de aplicación y análisis de vivencias socioemocionales en familias de jóvenes con Síndrome de Down

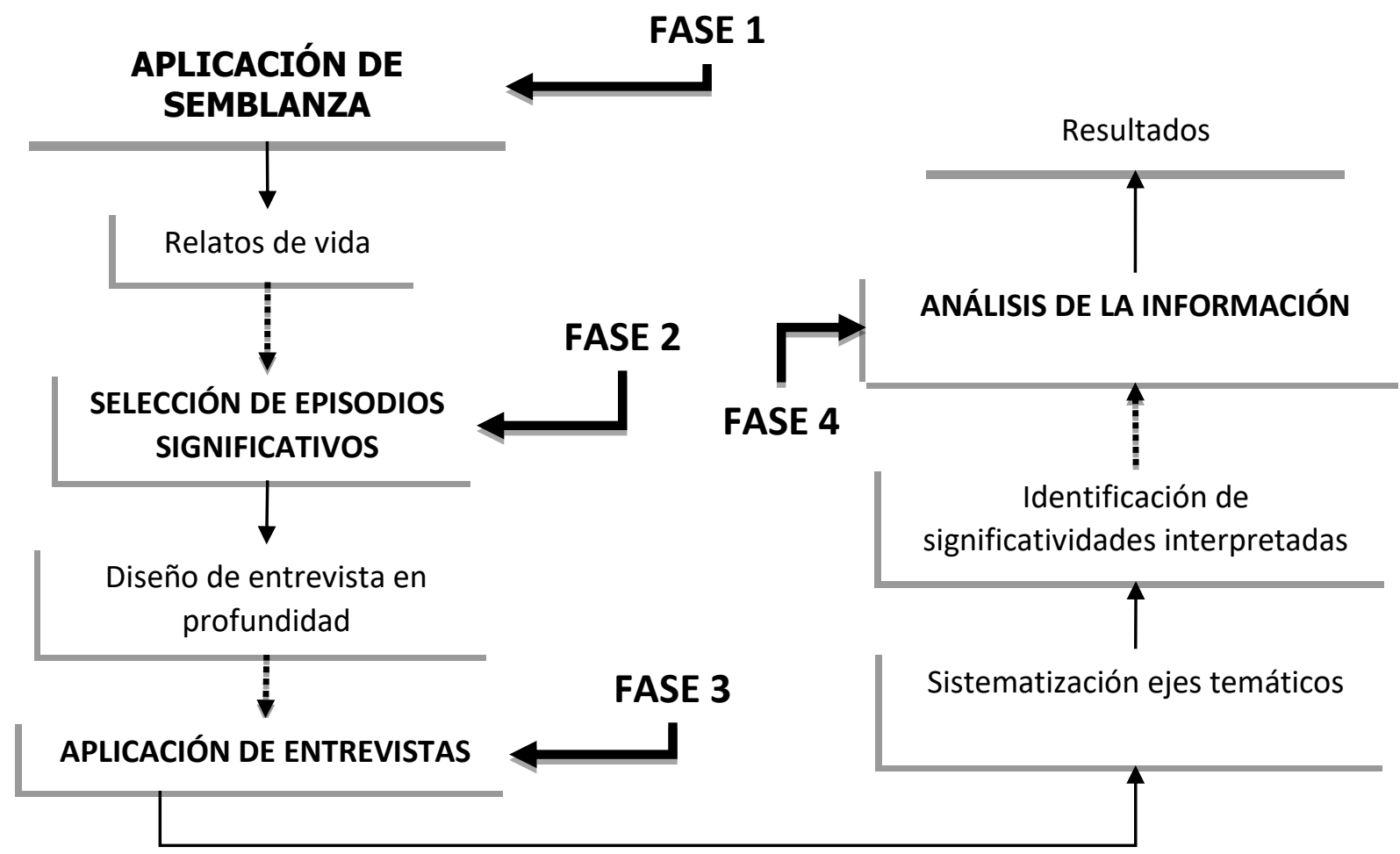

Fuente: Elaboración propia, 2018.

\subsection{Procedimientos}

El acceso al campo se realizó durante el primer semestre de 2018, y a través de la directiva de la Agrupación PLC Down, a la que se le comunicó la temática y objetivos de investigación. Posteriormente, en una reunión, se presenta el proyecto, se resuelven dudas y se invita a las familias a colaborar voluntariamente.

Precisamente, en cuanto a los criterios éticos, uno de los procedimientos es la entrega de consentimiento informado para conocimiento, aceptación o negación de participar en la investigación. De este modo, se declara la finalidad del estudio, el destino de la información recabada, así como el compromiso a entregar a cada familia un reporte de los resultados del estudio. De igual forma, el equipo de investigación se compromete a mantener la confidencialidad y anonimato de los datos obtenidos, mediante la sustitución del nombre quienes participaron. Adicionalmente, para presentar los testimonios, y al mismo tiempo resguardar la identidad de las personas, se estandariza el testimonio con una expresión alfanumérica (E1, E2, E3, E4 y E5) según cada entrevista realizada a las familias. 
La aplicación de los instrumentos se realizó entre octubre y noviembre de 2018, previa coordinación con las personas participantes. En este caso, padres y madres redactaron una semblanza en sus hogares. En cambio, la entrevista en profundidad fue realizada en dependencias de la Agrupación PLC Down.

El equipo de investigación transcribió digitalmente la semblanza para así asegurar la legibilidad del texto. Esta transcripción dio paso a la lectura e identificación de episodios significativos para las familias, y a partir de estos se elaboró un listado de preguntas para el diseño de la entrevista en profundidad.

Las entrevistas fueron registradas en audio digital para así sistematizar ejes temáticos e identificar significatividades interpretadas por quienes participaron. A partir de este procedimiento se transcriben relatos textuales que contienen las significatividades interpretadas. En efecto, “...el sujeto realiza acciones que están cargadas de significados. Todas sus acciones tienen un sentido; aunque el actor no haya tenido intención de significar algo, su acción puede ser interpretada por otro" (Rizo, 2007, p.3).

Finalmente, se exponen vivencias comunes entre quienes participaron del estudio, pero también se destaca la diversidad de situaciones experimentadas en donde las instituciones de educación, salud, y la familia contribuyen al bienestar o malestar socioemocional de padres y madres de hijos o hijas con SD.

\subsection{Análisis de datos}

El análisis de datos consideró un proceso inductivo-deductivo, mediante el cual el investigador o investigadora se mueve continuamente entre lo inductivo para desarrollar conceptos, categorías y relaciones (Flick, 2007; San Martín, 2014). En este caso, las categorías o temáticas (procedimiento deductivo) que forman parte de los resultados se construyeron desde la identificación de significatividades interpretativas (procedimiento inductivo). Es decir, no se consideró categorías analíticas pre-establecidas por teorías vinculadas a la temática. Por el contrario, la propia vivencia de las familias representó la fuente directa para la construcción categorial/temática.

Las significatividades interpretativas representan un recurso analítico que se deriva de la socio-fenomenología de Schütz (2003), y recientemente desarrollado como metodología de análisis para la vida social cotidiana (San Martín, 2018a; San Martín, 2018b; San Martín, 2018c). De este modo, se trata de una interpretación (mutua) de los códigos de acción de la comunidad y el actor. Es decir, actor y comunidad muestran modos de interpretar- 
comprender la realidad experimentada. Refieren a esquemas interpretativos que develan un orden intersubjetivo, que mediante la reproducción acentúa la realidad hasta convertirla en sentido común. En la práctica analítica, las significatividades interpretativas se identifican a partir de la descodificación de eventos, expresiones sociales y discursos cotidianos.

En esta investigación se empleó una triangulación de datos que, según Aguilar y Barroso (2015), es "la utilización de diferentes estrategias y fuentes de información sobre una recogida de datos que permite contrastar la información recabada" (p.74). Asimismo, es de carácter personal, ya que según el mismo autor se basa en diferentes muestras de sujetos.

Los criterios de rigor de la presente investigación son credibilidad, lo que se evidencia en la triangulación de datos. La consistencia a través de la validación de expertos y la descripción detallada del proceso de recogida, análisis e interpretación de los datos. Y por último la fidelidad de las transcripciones textuales de las entrevistas, contrastación de los resultados con la literatura existente y la identificación y descripción de limitaciones de la investigación (Noreña, Alcazar-Moreno, Rojas y Rebolledo-Malpica, 2012).

\section{Resultados}

Los resultados de la investigación se organizaron en cinco categorías relacionadas entre sí (Figura 2). En consecuencia, en este apartado se realiza una breve introducción para cada categoría, luego, se expone las significatividades interpretadas, y se finaliza con las vivencias expuestas por cada familia. 


\section{Figura 2}

Ejes temáticos para familias con hijos e hijas con Síndrome de Down de la comuna de Padre las Casas, Araucanía-Chile, 2019

Fuente: Elaboración propia, 2019.

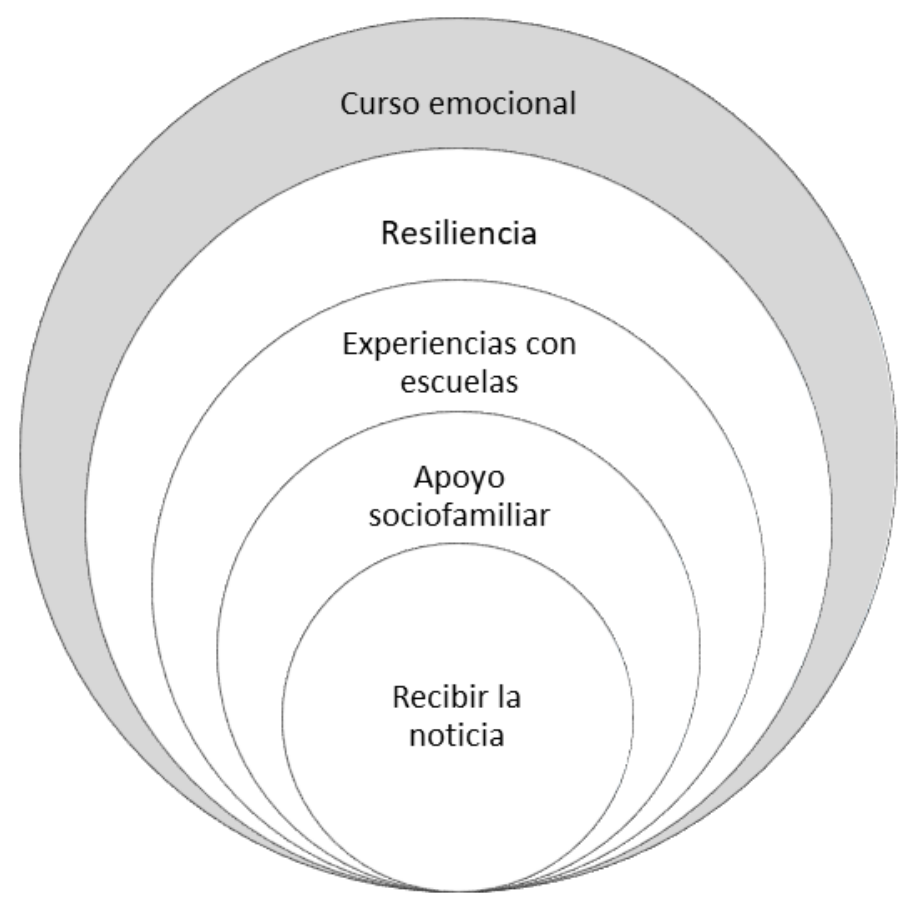

\subsection{Recibir la noticia}

El recibir la noticia del nacimiento de un hijo o una hija con discapacidad es uno de los primeros acontecimientos que marca la vida de las familias, desestabilizándolas y produciendo diversas reacciones emocionales.

\subsubsection{Significatividad interpretada. Fue difícil digerirlo}

Al conocer la noticia del diagnóstico de su hijo o hija, la familia recibe exceso información en relación con SD, lo que produce conmoción y falta de comprensión sobre el diagnóstico informado. Por tanto, se produce un estado de shock emocional que paraliza a padres y madres: "que te digan: oye, tu hijo es SD ¿qué es SD?. Si no teníamos idea de qué se trataba, fue difícil digerirlo en ese sentido" (E2). Esta noticia-diagnóstico representa el inicio de las vivencias socio-emocionales de las familias, por cuanto son recibidas de manera violenta, y sin considerar el estado de sensibilidad emocional de padres y madres de hijos o hijas con SD. 


\subsubsection{Significatividad interpretada. Esa fue la parte más cruel}

Los padres y madres expresan que el lenguaje utilizado por profesionales del área de salud para dar a conocer la condición de sus hijos e hijas no es pertinente, ya que utilizan términos de connotación negativa asociados a presencia y efectos del SD:

...su hijo se parece a alguien o tiene rasgos mongólicos [expresado por la profesional del área de salud]. Trágame tierra, me quería morir. Primero porque yo no sabía nada, yo había visto algún niñito por ahí, pero no tenía idea de lo que se trataba, o de lo que estábamos hablando, nadie se lo explica entonces, en ese momento uno tampoco, y lo único que hice fue llorar, lloré como tres meses (E4).

Esta situación produce un bloqueo emocional, donde se experimentan sentimientos de angustia y tristeza frente a estereotipos reproducidos por profesionales de la salud. En este caso, el mongolismo opera de manera violenta en un contexto de sensibilidad emocional de la familia. Tal como expresan las familias, recibir la noticia define un antes y un después en sus vidas, principalmente por el desconocimiento que tiene sobre la condición que profesionales informan. En las familias surgen una serie de pensamientos y emociones que se superponen, y que generan un escenario de incertidumbre que decanta en un estado de angustia permanente.

\subsection{Apoyo socio familiar}

La unidad familiar y el entorno social proveen de apoyo emocional, facilitan recursos para satisfacer necesidades que emergen durante la etapa de desarrollo de un hijo o hija con SD. Padres y madres interpretan este acompañamiento como esencial para enfrentar el proceso de tener un hijo o hija con SD.

\subsubsection{Significatividad interpretada. ...Se transformó ella en esa mamá}

Para algunas familias pertenecer a una agrupación se torna importante, ya que en la interacción con miembros de la agrupación se suplen carencias afectivas que en ocasiones no logran satisfacerse en el seno familiar:

...como que se transformó ella en esa mamá, en esa mamá que... Bueno, mi mamá, por ejemplo, nunca me fue a ver. Con todo este proceso mi mamá nunca me fue a ver, ni cuando nacieron nuestros hijos e hijas [...] pero igual uno, como que en el corazón está como resentida $(\mathrm{E} 1)$. 
La presencia de una persona que brinde contención emocional a un miembro de la familia afecta positivamente el estado de ánimo de la persona. En este sentido, personas ajenas a la familia se transforman en soportes emocionales para sobrellevar situaciones complejas.

\subsubsection{Significatividad interpretada. Alguien compartía la pena que yo tenía}

Frente a la necesidad de comprensión y de ser escuchado, los miembros de la familia recurren a personas que puedan entender la situación e intensidad de las emociones experimentadas. Por ejemplo, angustia, temor y desesperación ante el primer acercamiento del diagnóstico de su hijo o su hija:

...y mi mamá, me acuerdo que ella me abrazó, me dijo: no hijo, estas cosas...probablemente no es así [madre del entrevistado]. Y lloré, lloré todo lo que tenía que llorar, me desahogué como tenía que hacerlo. Entonces, eso fue como también, una sensación de sentir, no sé, que alguien compartía la pena que yo tenía en ese momento (E2).

Un aspecto positivo en la vida de las familias es contar con apoyo expresado en contención emocional. Este tipo de apoyo puede ser proporcionado por familiares directos, amigos, miembros de agrupaciones, y/o profesionales de las diversas áreas. Precisamente, el sentimiento ser escuchados por otras personas que han pasado por la misma experiencia, es una motivación para continuar el proceso de atención a sus hijos o hijas.

\subsection{Experiencias con escuelas}

Para cualquier familia escoger un establecimiento educacional que cumpla con sus expectativas educativas y de seguridad para su hijo o hija es una decisión importante y difícil de tomar. Más aún, cuando este hijo o hija requiere de apoyos profesionales acordes con sus necesidades.

\subsubsection{Significatividad interpretada. Me esperaban a mí solo para acusarme}

El profesorado debe mantener una constante comunicación con las familias para asegurar un vínculo estable. No obstante, existe una delgada línea entre comunicar y reprochar, ya que se tiende a dar mayor énfasis a aspectos negativos. Se invisibilizan dificultades que podrían estar viviendo. Así, padres y madres se sienten agotados, 
estresados, situación que sobrepasa sus límites emocionales. Esto en definitiva genera un distanciamiento con la escuela:

Me esperaban. ¿Sabe que su hijo botó la bandeja?, ¿sabe que su hijo tiró un escupo? ¿Usted cree que, de la casa, nosotros, con mi marido, nos escupimos? En la casa no hay ningún cabro chico, él lo está viendo aquí en el colegio. Háganse responsables ustedes aquí en el colegio. ¡Ya basta con lo que tengo en mi casa, aquí está el colegio!" (E1).

\subsubsection{Significatividad interpretada. Era mejor una escuela normal}

Las familias consideran que al principio existían dificultades para optar a otras opciones educativas, distintas de escuelas especiales. Si bien, estas escuelas especiales respondían a las necesidades de niños y niñas, para padres y madres una 'escuela normal', es la mejor opción: “....recién estábamos hablando de integración, una especial no es mala para ellos, pero era mejor una escuela normal, el problema: ¿dónde te abrían las puertas de la escuela regular?" (E4). Aunque actualmente el acceso a establecimientos educativos regulares ha mejorado, aún existen barreras en la cultura escolar. Estas barreras están relacionadas con prácticas a nivel profesional (actitudes, habilidades socioemocionales, competencias) como institucional (visión, misión). Esto ha provocado una ruptura del lazo familia-escuela, ya que la primera experimenta cuestionamientos hacia su rol parental.

\subsection{Resiliencia}

Un elemento común entre las familias participantes de este estudio es la capacidad de sobreponerse a situaciones difíciles. Esta capacidad les permite realizar acciones en beneficio de sus hijos e hijas, aun cuando no están exentas de experiencias frustrantes y dolorosas.

\subsubsection{Significatividad interpretada. Tenía que pararme por él}

En la experiencia de perder a uno de los padres o madres, la familia experimenta un estado de desequilibrio, dado que el integrante que se pierde representa estabilidad económica y emocional. De esta manera, se debe suplir el vacío que deja la pérdida, en un contexto emocional de tristeza y desamparo:

Mi hijo mayor tenía 16, él tenía 12 y el menor tenía 10. Yo no trabajaba tampoco, él era todo [referido a su esposo] y lloré harto. Todavía lloro, pero sé que me tenía que parar, 
porque tenía a mis hijos e hijas, tenía a mi mamá también y a mi hijo pequeño que era lo más importante. No quiero decir que los otros no sean importantes, pero tenía que pararme por él, fue demasiado triste, pero hemos salido adelante (E4).

\subsubsection{Significatividad Interpretada: El primer día la saqué para que todos la vean}

Cuando uno de los padres o madres experimenta una situación de discapacidad (física), esto resulta en una herramienta suficiente para empoderar a su hijo o hija. "Yo sufrí mucho cuando era niña... aprendí a caminar sola a los 8 años, mis papás ni siquiera me tomaban la mano. Pero yo era valiente, dije yo: no voy a quedar así" (E5). En este caso, la madre adquiere una actitud de seguridad frente a su discapacidad. Esto se traduce en una personalidad resiliente que favorece la vinculación temprana de su hija con la sociedad. En consecuencia, prevalece la aceptación de la discapacidad que nutre los lazos familiares. "Yo nunca voy a ser como mi madre, yo el primer día la saqué para que todas la vean. Jamás me preguntaron: ¡Uy pobrecita...! ¿Qué es lo que tiene?, nada” (E5). Este proceso les posibilita a las familias volver a encontrar en sí mismas los recursos necesarios para responder a las demandas de los contextos en que se desarrollan. A partir de esto logran estabilidad para proteger a todos los miembros de su familia.

\subsection{Curso emocional}

Las familias experimentan multiplicidad de emociones, positivas o negativas, que no son fijas y que surgen dependiendo de la situación por la que atraviesan en las distintas etapas de sus vidas y las de sus hijos e hijas.

\subsubsection{Significatividad interpretada. Me arrepiento todos los días de mi vida}

En principio, la familia experimenta pensamientos y sentimientos hostiles hacia su hijo o hija. Estos sentimientos se distancian con el vínculo natural entre padre/madre e hijo o hija, por cierto, es un sentimiento que altera la relación natural de afecto hacia la otra persona. Cabe señalar, que de acuerdo con la experiencia, en este caso de la madre, son las instituciones a través de los y las profesionales las que estimulan el sentimiento de rechazo hacia el hijo o hija con SD. Así, en esta experiencia la emocionalidad se torna negativa y se traduce en un shock emocional:

Tengo que ser sincera, yo decía, ojalá que se muera y pensaba en eso. Hablé con una psicóloga y le expliqué, me dijo que era normal que tú lo pensaras, porque la forma en 
que te dijeron es lo que te hirió [profesional de la salud]. $Y$ ahora me arrepiento todos los días de mi vida, pero ya en esos momentos no era yo (E4).

\subsubsection{Significatividad interpretada. Es mi razón de vivir}

Debido a las diferentes experiencias que atraviesan las familias y los lazos afectivos que construyen, para una abuela, su nieta ha sido motivo de grandes satisfacciones personales. Las vivencias socio-emocionales conectan a las familias con sentimientos primarios, en este caso con el amor hacia la nieta. Al mismo tiempo, este sentimiento de amor se traduce en un nuevo aliento para continuar el curso emocional que representa tener un hijo o hija con SD. En definitiva, la vida se resignifica, y permite movilizar nuevos sentimientos positivos, por ejemplo de orgullo:

Algo bonito, maravilloso, porque mi nieta es algo que a mí me hace feliz, verla así, yo le digo... Cuando yo digo, que le digo a mis hijos e hijas, por ejemplo, ¿saben que mi nieta me ha dado más satisfacciones que lo que me han dado ustedes tres juntos?. Mi nieta es mi razón de vivir (E3).

\subsubsection{Significatividad interpretada. Sé que tiene a sus hermanos}

Las familias suponen que el cuidado de sus hijos e hijas con SD será asumido por sus hermanos o hermanas, quienes se harán cargo cuando los padres y madres no estén. Si bien son conscientes que no tienen absoluta responsabilidad en el cuidado del hijo o hija con $\mathrm{SD}$, entienden que los hermanos o hermanas deberán asumir el rol de cuidado.: “...sé que tiene a sus hermanos y ellos lo van a cuidar, les tengo metido en la cabeza, pero igual entiendo de que no tengo por qué pasarles la responsabilidad a mis hijos e hijas, porque no es su responsabilidad" (E4). En efecto, las emociones son una constante en la vida de las familias, y se dan en una trayectoria durante el ciclo vital. Los sentimientos deben ser considerados como parte importante de la vida de todos los miembros del núcleo familiar, en la medida en que estos se encuentren en equilibrio, permitirán una mejor armonía en el hogar. A continuación, la Tabla 3 presenta una sistematización de los aspectos más relevantes de cada uno de los ejes temáticos. 
Tabla 3

Síntesis de los ejes temáticos y significatividades interpretadas en familias de hijos e hijas con SD de la comuna de Padre las Casas, Araucanía-Chile, 2018

\begin{tabular}{lll}
\hline Ejes temáticos & Aspectos centrales & Significatividades interpretadas \\
\hline Recibir la noticia & $\begin{array}{l}\text { Shock inicial frente al diagnóstico } \\
\text { Escaso conocimiento inicial sobre SD }\end{array}$ & Fue difícil digerirlo \\
Apoyo socio familiar & $\begin{array}{l}\text { Acompañamiento de agrupaciones } \\
\text { Contención emocional de familiares }\end{array}$ & Alguien compartía la pena que yo tenía \\
Experiencias con escuelas & $\begin{array}{l}\text { Vinculo limitado entre escuela-familia } \\
\text { Opciones educativas }\end{array}$ & Me esperaban a mí solo para acusarme \\
Resiliencia & $\begin{array}{l}\text { Herramientas para la transformación } \\
\text { Trayectoria emocional de las familias }\end{array}$ & Tenía que pararme por él \\
Curso emocional & Naturalización de rol de cuidador & Me arrepiento todos los días de mi vida \\
& Miedos hacia el futuro de hijos e hijas &
\end{tabular}

Fuente: Elaboración propia, 2019

\section{Discusión}

Los resultados sobre vivencias de las familias con hijos e hijas con SD dan cuenta de diversas situaciones socioemocionales que demuestran la fortaleza y determinación de la familia. Las vivencias normalmente son productos de relaciones sociales y/o vínculos con instituciones y organizaciones. En estas relaciones de apoyo, o en algunos casos de rechazo, se advierten sentimientos frente a la situación del hijo o hija con SD, redes de apoyo, presencia de la escuela, y capacidad de enfrentar las dificultades, que configuran el curso emocional de las familias. En efecto, la subjetividad_de padres y madres está determinada por las vivencias socio-emocionales producidas, no sólo por el hecho de tener un hijo o hija con SD, sino que por la relación y acompañamiento que las instituciones, organizaciones y comunidad en general pueden ofrecer a las familias.

\subsection{Sentimientos frente a la discapacidad}

Las vivencias que expresan las familias cuando se enteran del nacimiento de un hijo o una hija con SD provocan angustia, pena y desilusión frente a la situación vivida. Se produce un periodo de shock emocional que impide la comprensión de la información entregada por profesionales. Se trata de un acontecimiento impactante (Villa, Barrios y García, 2019), una etapa crítica (Villavicencio et al., 2018) que se caracteriza por la toma de conciencia parcial de la pérdida del bebé ideal (Rossel, 2004). Estas emociones preponderantes cambian, no son estáticas, tampoco tienen un orden determinado y su intensidad varía de manera 
considerable. Sin embargo, existen momentos de alegría debido a logros de sus hijos e hijas, esto proporciona satisfacción y se reconocen las contribuciones positivas de la familia (Villavicencio et al., 2018; Romero y Peralta, 2012; Rossel, 2004; Ruiz, et al., 2009).

Los hallazgos revelan sentimientos de dolor y sufrimiento por una escasa asertividad de los y las profesionales para comunicar la noticia del diagnóstico de SD a las familias. Bastidas y Alcaraz (2011) mencionan que los y las profesionales utilizan un lenguaje peyorativo. Esto provoca una visión pesimista de una discapacidad sin solución. Por tanto, recibir la noticia genera un desgaste emocional en las familias (Villavicencio et al., 2018). Se produce un sentimiento de vulnerabilidad. En palabras de Arellano y Peralta (2015), existe un rechazo hacia el cuerpo profesional que realizó el diagnóstico. Esta situación revela la importancia que adquieren las prácticas relacionales, específicamente el componente humano en las interacciones que se establecen entre los y las profesionales y las familias de niños y niñas con SD (Tapia et al., 2017).

Sumado a lo anterior, los planteamientos de Cunningham (2000) y Darezzo y Dupas (2011) señalan que la falta de información clara y un estilo profesional impersonal incrementa el rechazo y el descontento de las familias. Los resultados de esta investigación dejan en evidencia la necesidad de empatizar y acoger las emociones de las familias. Según Rossel (2004) se requiere que los equipos de salud neonatales comprendan y respeten las etapas por las que pasa la familia, para fomentar el vínculo madre-hijo o hija. De acuerdo con las experiencias vividas por las familias es un desafío desarrollar en los y las profesionales el componente humano (Tapia, et al., 2017) y, a su vez, contar con protocolos para comunicar la noticia (Azócar, Cerda y Mella, 2017).

Otro aspecto relevante es el temor de las familias sobre el futuro de sus hijos e hijas en situación de discapacidad. La angustia, estrés e incertidumbre del cuidado futuro del hijo o hija con SD se convierte en una reflexión constante, sobre todo al constatar que muchas veces la lucha se da individualmente. La literatura especializada señala que los hermanos y hermanas quienes serían los más idóneos como cuidadores futuros (Carrete, 2012; Flórez, 2016). Por esta razón, es importante construir vínculos colectivos y humanos que retroalimenten positivamente la esperanza y expectativas de las familias. Las redes de apoyo generadas a nivel local pueden alejar la idea de que es una lucha individual, la solidaridad y capacidad de escuchar, se traducen en valores necesarios que permiten enfrentar el miedo al futuro que expresan padres y madres de hijos e hijas con SD. 


\subsection{Apoyo indispensable frente a la adversidad}

Poseer algún tipo de apoyo es indispensable para las familias, ya sea de otros miembros o personas de alguna agrupación donde se consideren acogidos. Esto les permite alivianar la carga emocional que conlleva todo el proceso de adaptarse a la situación de tener un hijo o una hija con SD (Benítez, 2015; Darezzo y Dupas, 2011; España Down, 2012). Desde esta perspectiva, se evidencia un escenario adverso cuando estos apoyos se encuentran ausentes o pasivos, ya que las familias experimentan más estrés y cansancio, debido a que no pueden con todo lo que se les demanda. En consecuencia, requieren de apoyo socioemocional que atenúe aquellos sentimientos de aislamiento, y favorezca el tiempo personal para el descanso. Este apoyo socioemocional permitirá a las familias vivir sanamente, y sin tantas preocupaciones (Benítez, 2015; Villavicencio, et al., 2018).

Tanto las familias, como los y las profesionales tienen la responsabilidad de comenzar a generar cambios. En particular, se debe dar lugar a que la familia exprese sus necesidades, mediante la orientación de acciones que favorezcan y resguarden el bienestar personal (emocional) de padres y madres. Pero más allá del desempeño profesional, se debe apelar a un compromiso ético-profesional que sitúe a la familia y al hijo o hija con SD como centro de la responsabilidad ética-social de profesionales e instituciones. Probablemente, haga falta repensar el enfoque médico en el cual prima la racionalidad científica, porque precisamente en este enfoque el sujeto es reducido a objeto de estudio, y por tanto desprovisto de una experiencia socio-emocional.

\subsection{Realidades educativas}

El vínculo entre escuela y familia se ha ido debilitando, ya que se recriminan las prácticas que cada una lleva a cabo. De esta forma, en la escuela predomina un discurso que destaca aspectos negativos de los niños o niñas con SD. Entonces, es necesario que los y las profesionales retroalimenten fortalezas sobre falencias de niños y niñas con SD. Este hallazgo insta a la escuela a ser consciente y autocrítica respecto de la calidad del vínculo con las familias, así como de las responsabilidades y deberes que a cada uno le corresponden. Para evitar un escenario educativo adverso para las experiencias socioemocionales de las familias, es conveniente atender a enfoques actuales de atención temprana para robustecer el vínculo familia-escuela (Tapia, et al., 2017). Se requiere que cada participante se sienta considerado y acogido como parte de la comunidad educativa en que se fomente la colaboración, el compromiso y la confianza (Calvo, Verdugo y Amor, 
2016). La familia es un eslabón primordial en el proceso de apoyo, por tanto su participación y experiencia es un aporte a la simetría en el trabajo coordinado y colaborativo con equipos profesionales (Soto e Hinojo, 2004).

Se considera necesario remirar la labor profesional de interacción con familias. El propósito es generar lazos de colaboración que permitan apoyar de manera pertinente al estudiantado. Las personas profesionales están invitadas a activar fuertemente la empatía y desarrollar variadas estrategias para apoyar y empoderar a las familias, de manera que niños y niñas con SD se beneficien de las oportunidades para aprender y participar que ofrece la escuela.

\subsection{Superación de barreras}

Un común denominador entre las familias es la metáfora 'salir adelante', expresión que representa la complejidad de tener un hijo o una hija con SD. Para 'salir adelante' se despliegan habilidades que se desarrollan a través de la experiencia, y que permiten afrontar momentos críticos en el curso de vivencias. Por ejemplo, habilidades para orientar las emociones de modo que no afecten a otros integrantes de la familia. Al mismo tiempo, se reestructuran acciones que causan dolor emocional intenso (Naranjo, 2010; Ocampo y Vargas, 2018), esto ocurre en el caso de las relaciones sociales donde la familia se ve enfrentada al rechazo ya sea de las instituciones o de familiares. El maltrato de las instituciones muchas veces puede exponer a las personas a traumas que dificultan aún más su vida cotidiana. Es decir, las formas de relación, la información, el apoyo técnicoprofesional, deben estar al servicio de la justicia social, de manera que se minimice los episodios de maltrato.

\section{Conclusiones}

Las conclusiones presentadas a continuación resultan de un estudio de casos derivado de una investigación cualitativa, por tanto las conclusiones permiten comprender el contexto sociocultural en que se realizó la investigación. Entonces, en ningún caso se pretende generalizar y/o estandarizar la experiencia descrita, en su lugar, mediante el análisis de casos se constata la importancia de la familia en el desarrollo y crecimiento de los hijos e hijas es innegable. Rol que se ve amenazado cuando nace un niño o niña con SD. Esta situación desestabiliza la armonía familiar y social, y genera sentimientos ambivalentes en 
todos los miembros del hogar. De este modo, se vivencian etapas emocionales que obligan a la adaptación y reorganización de la familia a la nueva realidad.

Recibir la noticia de un hijo o hija con SD es el primer hecho que trastoca la sensibilidad del grupo familiar, por cuanto, el ideal de bebé y las proyecciones sobre él, se ponen en cuestionamiento. Se observa, que en el momento en que la familia recibe la noticia, comienza un curso o trayectoria emocional que acompaña a la familia por el resto de sus vidas. Pero este curso emocional también se complementa con un escenario social expresado por la relación que la familia emprende con instituciones y organizaciones que acompañan el proceso de tener un hijo o hija con SD. Por lo cual, el apoyo social se instala como un aspecto imprescindible en la vida de las familias, principalmente porque padres y madres buscan un espacio para sentirse comprendidos y acompañados por personas que con vivencias similares.

Las etapas emocionales que se viven en cada una de las experiencias de las familias, condicionan el rumbo que tomarán frente a la discapacidad de su hijo o su hija. Y, aunque, estas no necesariamente se afrontan de manera lineal, presentándose a lo largo del ciclo vital; impacto, negación, tristeza o dolor, adaptación y reorganización, cada una de ella se caracteriza por manifestar claros sentimientos que experimentan miembros del núcleo familiar. En particular, un aspecto común de las familias es la resiliencia, que permite el aprendizaje de herramientas necesarias para que los hijos e hijas logren autonomía y la mejor calidad de vida posible. En este sentido, es posible señalar que la fortaleza emocional de padres y madres no surge de modo inmediata apenas conocen la noticia que diagnóstica al hijo o hija con SD, por el contrario el apoyo social expresado en el acompañamiento de organizaciones, comunidad y algunas instituciones, contribuyen al desarrollo de la resiliencia.

Respecto de las limitaciones del estudio, se advierte como necesario incorporar evidencias narrativas a partir de las semblanzas realizadas. En este sentido es conveniente proponer metodologías que permitan rescatar y destacar la riqueza de la semblanza como una técnica que otorga autonomía a las personas participantes del estudio. Sobre todo porque la semblanza en tanto técnica autobiográfica permite expresar las vivencias socioemocionales que tienen sentido para los padres y madres. Así, el escenario biográfico permite comprender la situación social y emocional de las familias que es donde se articulan empíricamente las significatividades interpretadas.

Se sugieren que las políticas públicas permitan la formación de profesionales centrados en la familia y en sus necesidades físicas, psicológicas y sociales. Precisamente, 
esta investigación sirve como un recurso para que las instituciones de formación reformulen protocolos de comunicación y relación que tienen con las familias. El uso de aquellos estereotipos asociados al SD que pueden dañar la sensibilidad de padres y madres, deben ser abordados como una temática relevante en la actuación profesional. Esto implica reposicionar a la familia y a los hijos e hijas con SD como sujetos con historia, que sienten e interactúan a partir de su vivencia como padres y madres con hijos e hijas diferentes, en consecuencia con necesidades de aprender de profesionales comprometidos ética y socialmente.

\section{Agradecimientos}

Para la realización de esta investigación resultó indispensable la cooperación y disposición de las familias pertenecientes a la agrupación PLC DOWN, por lo que se les agradece su apertura al develar aspectos íntimos de su realidad, que dan cuenta de sus historias de vida.

\section{Referencias}

Abramovich, Víctor. (2006). Una aproximación al enfoque de derechos en las estrategias y políticas de desarrollo. Revista de la CEPAL, (88). Recuperado de https://www.cepal.org/es/publicaciones/11102-aproximacion-al-enfoque-derechosestrategias-politicas-desarrollo

Aguilar, Sonia. y Barroso, Julio. (2015). La triangulación de datos como estrategia en investigación educativa. Pixel-bit. Revista de medios y educación, (47), 73-88.

Arellano, Araceli. y Peralta, Feli. (2015). El Enfoque centrado en la familia, en el campo de la discapacidad intelectual ¿Cómo perciben los padres su relación con los padres? Revista de Investigación educativa Rie, 33(1), 119-132.

Azócar, Elizabeth., Cerda, Jasmín. y Mella, Maribel. (2017). Experiencias en madres con hijos con síndrome de Down en torno a la recepción del diagnóstico. Revista Chilena de Salud Pública, 21(2), p. 113-122.

Bastidas, Miriam. y Alcaraz, Gloria. (2011) Comunicación de la noticia del nacimiento de un niño o niña con Síndrome de Down: el efecto de una predicción desalentadora. Rev. Fac. Nac. Salud Pública, 29(1), 18-24.

Benítez, Tatiana. (2015). El síndrome de Down desde la percepción familiar (Trabajo de fin de grado de Trabajo Social). Universidad de La Laguna, España. Recuperado el de https://riull.ull.es/xmlui/bitstream/handle/915/1031/EL\%20SINDROME\%20DE\%20DOW N\%20DESDE\%20LA\%20PERCEPCION\%20FAMILIAR.pdf?sequence=1 
Blasco, Josefa. y Pérez, José. (2007). Metodologías de investigación en educación física y deportes: ampliando horizontes. Recuperado de http://rua.ua.es/dspace/handle/10045/12270\#vpreview

Calvo, Mํㅡㄹ Isabel., Verdugo, Miguel Ángel., y Amor, Antonio Manuel. (2016). La Participación Familiar es un Requisito Imprescindible para una Escuela Inclusiva. Revista latinoamericana de educación inclusiva, 10(1), 99-113.

Carrete, Paula. (2012). Actualización: Familias con un miembro con discapacidad. Evidencia Actualización en la Práctica Ambulatoria, 15(1). Recuperado de http://repositoriocdpd.net:8080/handle/123456789/1005

Cunningham, Cliff. (2000). Familias de niños con Síndrome de Down. En Miguel Ángel Verdugo (ed), Familias y discapacidad intelectual. Madrid: FEAPS.

Darezzo, Michelle. y Dupas, Giselle. (2011). Independencia del niño con Síndrome de Down: la experiencia de la familia. Rev. Latino-Am. Enfermagem, 19(4), 985-993.

Down España. (2012). Apoyos y marcos de colaboración. Recuperado el 10 de junio del 2018 de http://www.sindromedown.net/wp-content/uploads/2016/07/III-Plan-deAccion-1.pdf

Flick. Uwe. (2007). Introducción a la Investigación cualitativa. España: Morata.

Flórez, Jesús. (2016). El Síndrome de Down en perspectiva 2016, Fundación Síndrome de Down de Cantabria. Recuperado de http://revistadown.downcantabria.com/wpcontent/uploads/2016/03/revista128 16-23.pdf

Giné, Climent., Gràcia, Marta., Vilaseca, Rosa. y Balcells-Balcells, Anna. (2009). Trabajar con las familias en atención temprana. Revista interuniversitaria de formación del profesorado, (65), 95-116.

Grau Rubio, Claudia. (2013). Fomentar la resiliencia en familias con enfermedades crónicas pediátricas. Revista Española de Discapacidad, 1(1), 195-212.

Hodapp, Robert. (2008). Familias de las personas con síndrome de Down: perspectivas, hallazgos, investigación y necesidades. Revista Síndrome de Down, 25, 17-32.

Huerta, Yolanda. y Rivera, María Elena. (2017). Resiliencia, recursos familiares y espirituales en cuidadores de niños con discapacidad. Journal of Behavior, Health \& Social Issues, 9(2), 70-81.

McWilliam, Robin. (2016). Metanoia en Atención Temprana: Transformación a un Enfoque Centrado en la Familia. Revista Latinoamericana de Educación Inclusiva, 10(1), 133153.

Ministerio de educación (2009). Ley General de Educación 20.370. Santiago, Chile. Recuperado

de http://www.leychile.cl/Consulta/m/norma plana?idNorma=1014974\&org= 
Ministerio de educación (2015). Ley 20.845 de inclusión escolar que regula la admisión de los y las estudiantes, elimina el financiamiento compartido y prohíbe el lucro en establecimientos educacionales que reciben aportes del estado. Recuperado de http://bcn.cl/1uv1u

Naranjo, Rolando. (2010). Desastres y resiliencia. Enfoque de las bases neurales de la tenacidad cognitiva, sintomatología nerviosa y conducta general a seguir. Rev. Hosp. Psiquiátrico de la Habana, 7(3). Recuperado de http://www.revistahph.sld.cu/hph0310/hph12310.html

Noreña, Ana., Alcazar-Moreno, Noemí., Rojas, Juan. y Rebolledo-Malpica, Dinora. (2012). Aplicabilidad de los criterios de rigor y éticos en la investigación cualitativa. Aquichan, 12(3), 263-274.

Ocampo, Liliana. y Vargas, Fabiana. (2018). Resiliencia en familias de personas con Síndrome de Down en San Pedro del Paraná, Paraguay. Revista científica e investigaciones, 6, 10-132.

Organización Naciones Unidas. (2006). Convención sobre los Derechos de las Personas con Discapacidad. Recuperado https://www.ohchr.org/Documents/Publications/AdvocacyTool sp.pdf

Otzen, Tamara. y Manterola, Carlos. (2017). Técnicas de Muestreo sobre una Población a Estudio. International Journal of Morphology, 35(1), 227-232.

Pujadas, Juan. (2002): El método biográfico: El uso de las historias de vida en ciencias sociales. Madrid: CIS.

Rizo, Marta. (2007). Intersubjetividad, comunicación e interacción. Los aportes de Alfred Schütz a la Comunicología. Razón y palabra, 12(57). Recuperado de http://www.redalyc.org/articulo.oa?id=199520710007

Romero, Cecilia. y Peralta, Sara. (2012). Estudio de la dinámica en familias con hijos e hijas/as con síndrome de Down. Eureka (Asunción) en Línea, 9(1), 69-77.

Rossel, Katherine. (2004). Apego y vinculación en el Síndrome de Down. Una emergencia afectiva. Revista Pediatría Electrónica, 1(1), 3-8.

Ruiz, Emilio., Álvarez, Raquel., Arce, Almudena., Palazuelos, Irene. y Schelstraete, Gunilde. (2009). Programa de educación emocional. Aplicación Práctica de niños con Síndrome de Down. Revista Síndrome de Down, 26, 126-140.

Ruiz, José. (2012). Metodología de la investigación cualitativa (Vol. 15). Bilbao: Universidad de Deusto.

Saavedra, Teresa. (2005). La Hermenéutica Reflexiva en la Investigación Educacional. Revista Enfoques Educacionales, 7(1), 51-66. 
San Martín, Daniel. (2014). Teoría fundamentada y Atlas.Ti: recursos metodológicos para la investigación educativa. Revista Electrónica de Investigación Educativa, 16(1), 104122. Recuperado de http://redie.uabc.mx/vol16no1/contenido-sanmartin.html

San Martín, Daniel. (2018a). ¿Artesanía o cazador tras la huella?. Reflexiones para el análisis cualitativo de datos. Empiria. Revista de metodología de ciencias sociales, (40), 65-83. doi:https://doi.org/10.5944/empiria.40.2018.22011

San Martín, Daniel. (Agosto 2018b). Propuesta metodológica para el análisis de la vida cotidiana en contextos escolares rurales. En N. Fernández Lamarra (Presidencia). VIII Congreso Iberoamericano de Pedagogía (CIP 2018). "La innovación y el futuro de la educación para un mundo plural”. Buenos Aires, Argentina.

San Martín, D. (Octubre 2018c). Tipificaciones en la vida social cotidiana de profesores de escuelas de campo. En J. Mansilla (Presidencia). XII Congreso Internacional de la Sociedad de Historia de La Educación Latinoamericana (Shela). Temuco, Chile.

Schutz, Alfred. (2003). El problema de la realidad social. Escritos I. Buenos Aires: Amorrortu.

Sistema de Protección Integral a la Infancia del Estado de Chile (2009). Chile Crece Contigo. Recuperado de http://www.crececontigo.gob.cl/acerca-de-chcc/que-es/

Simons, Helen. (2011). El estudio de Caso: Teoría y práctica. Madrid, España: Editorial, Morata.

Soto, Ronald. e Hinojo, Francisco. (2004). La colaboración entre maestros/maestras y padres/madres para atender a la diversidad en las instituciones educativas. Revista Educación, 28(2), 185-201.

Tapia, Carmen Paz., Palma, Andrea. y González, Karla. (2017). Atención temprana, percepción de madres de niños y niñas con síndrome de Down. Revista Actualidades Investigativas en Educación, 17(1), 1-22.

Urbano, Claudio. y Yuni, José. (2008). La discapacidad en la escena familiar. Córdoba, Argentina: Editorial Brujas.

Villa, Jenifer., Barrios, Sara. y García, Paula. (2019). Vivencia de cuidadoras principales de niños y adolescentes con necesidades especiales de atención en salud. Enfermería Comunitaria, 15. Recuperado de http://ciberindex.com/c/ec/e12067

Villavicencio, Carmita., Romero, Marcos., Criollo, Marco. y Peñaloza, Wilson. (2018). Discapacidad y familia: Desgaste emocional. Academo (Asunción), 5(1), 89-98. 
Revista indizada en

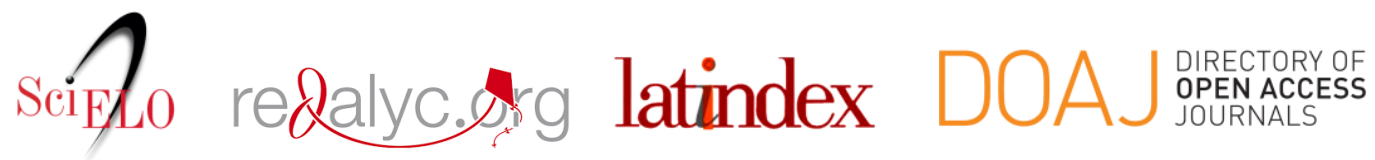

Distribuida en las bases de datos:

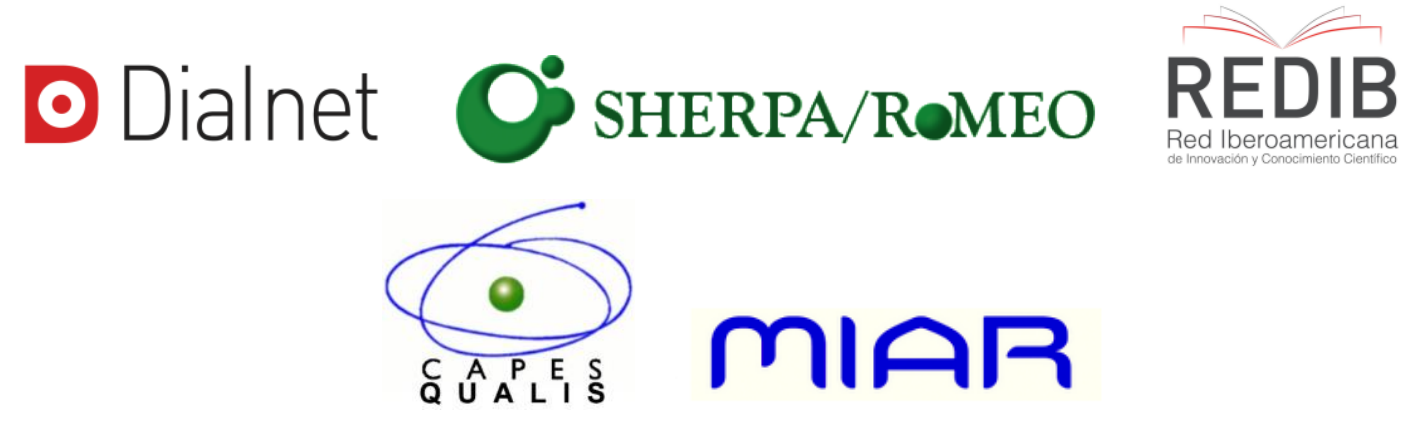

